\title{
General conditions for self-cancellation of geometric aberrations in a lattice structure
}

\author{
G. $\mathrm{Xu}$ \\ Institute of High Energy Physics, Yuquan Road 19(B), Beijing 100049, China \\ (Received 5 August 2005; published 24 October 2005)
}

\begin{abstract}
In this paper we will discuss the general conditions for self-cancellation of geometric aberrations in an uncoupled or even coupled lattice structure. The self-cancellation conditions show that $-I$ transformer even noninterleaved multipoles is not necessary. We will also give two examples for sextupole case to specify the application.
\end{abstract}

DOI: 10.1103/PhysRevSTAB.8.104002

PACS numbers: 29.27.Bd, 29.27.Eg, 41.85.Ja

\section{INTRODUCTION}

The design of lattice of an accelerator is for an onmomentum particle. Unfortunately, due to the energy spread, most of the particles in the accelerator are offmomentum. Because of the chromatic effects in the accelerator, many parameters of off-momentum particles such as tunes, beta functions and dispersions are different from the design values. The chromatic effect must be corrected or the beam will be degraded even unstable. The classical method of chromatic correction is to place the interleaved sextupoles at the locations where the dispersion is not zero. Since the achromat concept has been proposed in [1], the noninterleaved sextupole schemes have been applied for not only large storage ring [2-5], but also linear collider [6-9]. All of these noninterleaved sextupole schemes are to use $-I$ transformer in both $x$ and $y$ planes. In this paper we will show that $-I$ transformer is not necessary. In Sec. II we will introduce the transfer map of a pure multipole. In Sec. III, we will show some different sets of multipoles and their necessary conditions. In Sec. IV, we will discuss the case which the lattice has a strong $x-y$ coupling. In Sec. V, we will give two specific applications.

\section{TRANSFER MAP OF MULTIPOLE}

When a particle passes through a $2(m+1)$ th multipole with integral strength $k_{m}$ and rotating angle $\theta_{m}$, it will get a kick. Using vector $\left(x, x^{\prime}, y, y^{\prime}\right)$ to describe the particle position in $4 \mathrm{D}$ phase space, then after the kick,

$$
\begin{array}{ll}
x_{1}=x_{0}, & x_{1}^{\prime}=x_{0}^{\prime}+\frac{k_{m}}{m !} f_{m}\left(\theta_{m}, x_{0}, y_{0}\right), \\
y_{1}=y_{0}, & y_{1}^{\prime}=y_{0}^{\prime}+\frac{k_{m}}{m !} g_{m}\left(\theta_{m}, x_{0}, y_{0}\right),
\end{array}
$$

where $\left(x_{0}, x_{0}^{\prime}, y_{0}, y_{0}^{\prime}\right)$ and $\left(x_{1}, x_{1}^{\prime}, y_{1}, y_{1}^{\prime}\right)$ denoted by $\vec{X}_{0}$ and $\vec{X}_{1}$ are the positions before and after the multipole kick, respectively. And, the functions $f_{m}\left(\theta_{m}, x_{0}, y_{0}\right)$ and

$$
\begin{aligned}
g_{m}\left(\theta_{m}, x_{0}, y_{0}\right) \text { are defined as } & \\
f_{m}\left(\theta_{m}, x, y\right)= & \operatorname{Im}\left[(x+i y)^{m}\right] \sin (m+1) \theta_{m} \\
& -\operatorname{Re}\left[(x+i y)^{m}\right] \cos (m+1) \theta_{m}, \\
g_{m}\left(\theta_{m}, x, y\right)= & \operatorname{Im}\left[(x+i y)^{m}\right] \cos (m+1) \theta_{m} \\
& +\operatorname{Re}\left[(x+i y)^{m}\right] \sin (m+1) \theta_{m} .
\end{aligned}
$$

Equation (1) is the transfer map of a pure multipole with a rotating angle. Shortly, it can be denoted by

$$
\vec{X}_{1}=F\left(m, \theta_{m}, k_{m}, \vec{X}_{0}\right) .
$$

\section{DIFFERENT SETS OF MULTIPOLES FOR UNCOUPLED LATTICE}

\section{A. Set of two sextupoles}

Considering a particle passing through a $S_{1}-T_{12}-S_{2}$ channel, where $S_{1}$ and $S_{2}$ are two regular sextupoles with strength $k_{21}$ and $k_{22}, T_{12}$ is the transfer matrix between $S_{1}$ and $S_{2}$ as follows:

$$
T_{12}=\left(\begin{array}{cccc}
t_{11} & t_{12} & 0 & 0 \\
t_{21} & t_{22} & 0 & 0 \\
0 & 0 & t_{33} & t_{34} \\
0 & 0 & t_{43} & t_{44}
\end{array}\right)
$$

After passing through the channel, the position of the particle is

$$
\vec{X}_{1}=F\left[2,0, k_{22}, T_{12} F\left(2,0, k_{21}, \vec{X}_{0}\right)\right] .
$$

Expanding Eq. (5), one can get

$$
\begin{aligned}
& x_{1}=t_{11} x_{0}+t_{12} x_{0}^{\prime}+\frac{1}{2} k_{21} t_{12}\left(-x_{0}^{2}+y_{0}^{2}\right), \\
& y_{1}=t_{33} y_{0}+t_{34} y_{0}^{\prime}+k_{21} t_{34} x_{0} y_{0} .
\end{aligned}
$$

In order to eliminate the nonlinear terms in Eq. (6), $t_{12}$ and $t_{34}$ must be zero, i.e.,

$$
t_{12}=0, \quad t_{34}=0 .
$$


Further,

$$
\begin{aligned}
x_{1}^{\prime}= & t_{21} x_{0}+t_{22} x_{0}^{\prime}-\frac{1}{2}\left(k_{21} t_{22}+k_{22} t_{11}^{2}\right) x_{0}^{2} \\
& +\frac{1}{2}\left(k_{21} t_{22}+k_{22} t_{33}^{2}\right) y_{0}^{2} .
\end{aligned}
$$

Let the coefficients of $x_{0}^{2}$ and $y_{0}^{2}$ be 0 , one can get

$$
k_{21}=-k_{22} t_{11}^{3}, \quad t_{11}= \pm t_{33} .
$$

Note that we have used the unity condition of $T_{12}$, i.e.,

$$
t_{11} t_{22}=1, \quad t_{33} t_{44}=1 .
$$

Under the conditions of Eqs. (7) and (9),

$$
y_{1}^{\prime}=t_{43} y_{0}+t_{44} y_{0}^{\prime} .
$$

Equation (5) will be reduced to

$$
\vec{X}_{1}=T_{12} \vec{X}_{0}
$$

Equation (12) means the nonlinearity of the channel $S_{1}-T_{12}-S_{2}$ has been eliminated under the conditions of Eqs. (7) and (9). In terms of Twiss parameters at $S_{1}$ and $S_{2}$ and their phase advance, one can have

$$
\begin{aligned}
t_{11} & =\sqrt{\frac{\beta_{x 2}}{\beta_{x 1}}}\left(\cos \phi_{x 12}+\alpha_{x 1} \sin \phi_{x 12}\right), \\
t_{12} & =\sqrt{\beta_{x 1} \beta_{x 2}} \sin \phi_{x 12}, \\
t_{33} & =\sqrt{\frac{\beta_{y 2}}{\beta_{y 1}}}\left(\cos \phi_{y 12}+\alpha_{y 1} \sin \phi_{y 12}\right), \\
t_{34} & =\sqrt{\beta_{y 1} \beta_{y 2}} \sin \phi_{y 12} .
\end{aligned}
$$

From Eq. (7) one can get

$$
\phi_{x 12}=n_{x} \pi, \quad \phi_{y 12}=n_{y} \pi, \quad n_{x}, n_{y} \in \text { integer. }
$$

From Eq. (9), one can get

$$
\beta_{y 1} / \beta_{x 1}=\beta_{y 2} / \beta_{x 2}
$$

and

$$
k_{21} \beta_{x 1}^{3 / 2}=-(-1)^{n_{x}} k_{22} \beta_{x 2}^{3 / 2} .
$$

From Eqs. (14)-(16) one can see that the selfcancellation condition is independent from the $\alpha$ functions at $S_{1}$ and $S_{2}$.

The $-I$ transformer is corresponding to the following condition which is a special case of the Eqs. (14)-(16).

$$
\begin{aligned}
\beta_{x 1} & =\beta_{x 2}, \alpha_{x 1}=\alpha_{x 2}, \beta_{y 1}=\beta_{y 2}, \alpha_{y 1}=\alpha_{y 2}, \\
k_{21} & =k_{22}, \\
\phi_{x 12} & =n_{x} \pi, \phi_{y 12}=n_{y} \pi, n_{x}, n_{y} \in \text { odd. }
\end{aligned}
$$

Considering the chromaticity contribution, the signs of dispersions at two sextupoles must be identical for odd $n_{x}$ or opposite for even $n_{x}$, otherwise the net chromaticity contribution will decrease.

\section{B. Set of two multipoles}

For a set of two regular $2(m+1)$ th multipoles, the selfcancellation condition Eqs. (14) and (15) are still valid, but Eq. (16) will become

$$
k_{m 1} \beta_{x 1}^{(m+1) / 2}=-(-1)^{n_{x}} k_{m 2} \beta_{x 2}^{(m+1) / 2}
$$

Actually, for the hybrid of multipoles the selfcancellation conditions Eqs. (14) and (15) are also valid. The integral strength for each multipole component must satisfy Eq. (18). An additional condition is that the rotating angle of each multipole component for the two magnets must satisfy

$$
\theta_{m 2}-(-1)^{n_{x}+n_{y}} \theta_{m 1}=\frac{j_{m}}{m+1} \pi, \quad j_{m} \in \text { integer. }
$$

For the pure skew multipoles $\left(\theta_{m 1}=\theta_{m 2}=\{\pi /[2(m+\right.$ $1)]\})$, the integral strength of two multipoles must satisfy

$$
k_{m 1} \beta_{x 1}^{(m+1) / 2}=-(-1)^{n_{y}} k_{m 2} \beta_{x 2}^{(m+1) / 2}
$$

Comparing with Eqs. (18) and (20), one can see that the relative sign of strength of two regular multipoles is dependent on the horizontal phase advance while the relative sign of strength of two skew multipoles is dependent on the vertical phase advance. In fact, $k_{m}$ in Eq. (18) includes regular and skew components, but $k_{m}$ in Eq. (20) only includes skew component.

\section{Set of three sextupoles}

In $S_{1}-T_{12}-S_{2}$ channel, the self-cancellation condition requires the $\beta$ function at sextupoles $S_{1}$ and $S_{2}$ satisfying Eq. (15). In order to relax this condition, let us consider a $S_{1}-T_{12}-S_{2}-T_{23}-S_{3}$ channel. The position after passing through this channel is

$$
\vec{X}_{1}=F\left\{2, \theta_{3}, k_{23}, T_{23} F\left[2, \theta_{2}, k_{22}, T_{12} F\left(2, \theta_{1}, k_{21}, \vec{X}_{0}\right)\right]\right\},
$$

where $T_{12}$ and $T_{23}$ are the transfer matrix from $S_{1}$ and $S_{2}$ to $S_{2}$ and $S_{3}$, respectively. The horizontal and vertical phase advance of $S_{1}-S_{2}$ and $S_{2}-S_{3}$ must satisfy

$$
\begin{aligned}
& \phi_{x 12}=n_{x 1} \pi, \phi_{y 12}=n_{y 1} \pi \\
& \phi_{x 23}=n_{x 2} \pi, \phi_{y 23}=n_{y 2} \pi \quad n_{x 1}, n_{y 1}, n_{x 2}, n_{y 2} \in \text { integer, }
\end{aligned}
$$


and the strength of $S_{1}, S_{2}$, and $S_{3}$ must satisfy

$$
\begin{aligned}
& (-1)^{n_{x 1}} k_{21} \beta_{x 1}^{3 / 2} /\left(\frac{\beta_{y 3}}{\beta_{x 3}}-\frac{\beta_{y 2}}{\beta_{x 2}}\right) \cos 3 \theta_{1} \\
& =k_{22} \beta_{x 2}^{3 / 2} /\left(\frac{\beta_{y 1}}{\beta_{x 1}}-\frac{\beta_{y 3}}{\beta_{x 3}}\right) \cos 3 \theta_{2} \\
& =(-1)^{n_{x 2}} k_{23} \beta_{x 3}^{3 / 2} /\left(\frac{\beta_{y 2}}{\beta_{x 2}}-\frac{\beta_{y 1}}{\beta_{x 1}}\right) \cos 3 \theta_{3} .
\end{aligned}
$$

The rotating angle of the three sextupoles must satisfy

$$
\begin{aligned}
(-1)^{n_{x 1}+n_{y 1}} \frac{\sqrt{\beta_{y 1}}}{\sqrt{\beta_{x 1}}} \tan 3 \theta_{1} & =\frac{\sqrt{\beta_{y 2}}}{\sqrt{\beta_{x 2}}} \tan 3 \theta_{2} \\
& =(-1)^{n_{x 2}+n_{y 2}} \frac{\sqrt{\beta_{y 3}}}{\sqrt{\beta_{x 3}}} \tan 3 \theta_{3} .
\end{aligned}
$$

Under the conditions of Eqs. (22)-(24), Eq. (21) will be reduced to

$$
\vec{X}_{1}=T_{23} T_{12} \vec{X}_{0}
$$

Again, the nonlinearity of the channel $S_{1}-T_{12}-S_{2}-T_{23}-S_{3}$ has been eliminated.

If $\theta_{1}=\theta_{2}=\theta_{3}=\frac{\pi}{6}$, Eq. (23) will become

$$
\begin{aligned}
& (-1)^{n_{y 1}} k_{21} \beta_{y 1}^{3 / 2} /\left(\frac{\beta_{x 3}}{\beta_{y 3}}-\frac{\beta_{x 2}}{\beta_{y 2}}\right) \\
& =k_{22} \beta_{y 2}^{3 / 2} /\left(\frac{\beta_{x 1}}{\beta_{y 1}}-\frac{\beta_{x 3}}{\beta_{y 3}}\right) \\
& =(-1)^{n_{y 2}} k_{23} \beta_{y 3}^{3 / 2} /\left(\frac{\beta_{x 2}}{\beta_{y 2}}-\frac{\beta_{x 1}}{\beta_{y 1}}\right) .
\end{aligned}
$$

\section{Set of higher order multipoles}

To relax the condition of Eq. (15), in Sec. III C we use more than two sextupoles to make a self-cancellation
TABLE I. Number of multipoles for a self-cancellation channel.

\begin{tabular}{lcc}
\hline \hline Multipole & Number of RM & Number of SM \\
\hline 6 & 3 & 3 \\
8 & 4 & 3 \\
10 & 4 & 4 \\
12 & 5 & 4 \\
14 & 5 & 5 \\
16 & 6 & 5 \\
\hline \hline
\end{tabular}

channel. This way can be generalized to the higher multipole case. Generally speaking, for $2(m+1)$ th regular multipoles (RM), one need $[(m-1) / 2]+3$ multipoles to make such a channel, while for skew multipoles (SM), one need $[\mathrm{m} / 2]+2$ multipoles $([x]$ means the maximum integer less than $x$ ). We list the numbers for up to 16 th multipole in Table I.

In general, the multipoles can have some rotating angle, the relation among the strength and rotating angle of the different multipoles will be complicated. For octupole case, one can have the following self-cancellation condition

$$
\begin{aligned}
& k_{31} \cos 4 \theta_{1} \beta_{x 1}^{2}\left(\frac{\beta_{y 1}}{\beta_{x 1}}-\frac{\beta_{y 2}}{\beta_{x 2}}\right)\left(\frac{\beta_{y 1}}{\beta_{x 1}}-\frac{\beta_{y 3}}{\beta_{x 3}}\right)\left(\frac{\beta_{y 1}}{\beta_{x 1}}-\frac{\beta_{y 4}}{\beta_{x 4}}\right) \\
& =k_{32} \cos 4 \theta_{2} \beta_{x 2}^{2}\left(\frac{\beta_{y 2}}{\beta_{x 2}}-\frac{\beta_{y 1}}{\beta_{x 1}}\right)\left(\frac{\beta_{y 2}}{\beta_{x 2}}-\frac{\beta_{y 3}}{\beta_{x 3}}\right)\left(\frac{\beta_{y 2}}{\beta_{x 2}}-\frac{\beta_{y 4}}{\beta_{x 4}}\right) \\
& =k_{33} \cos 4 \theta_{3} \beta_{x 3}^{2}\left(\frac{\beta_{y 3}}{\beta_{x 3}}-\frac{\beta_{y 1}}{\beta_{x 1}}\right)\left(\frac{\beta_{y 3}}{\beta_{x 3}}-\frac{\beta_{y 2}}{\beta_{x 2}}\right)\left(\frac{\beta_{y 3}}{\beta_{x 3}}-\frac{\beta_{y 4}}{\beta_{x 4}}\right) \\
& =k_{34} \cos 4 \theta_{4} \beta_{x 4}^{2}\left(\frac{\beta_{y 4}}{\beta_{x 4}}-\frac{\beta_{y 1}}{\beta_{x 1}}\right)\left(\frac{\beta_{y 4}}{\beta_{x 4}}-\frac{\beta_{y 2}}{\beta_{x 2}}\right)\left(\frac{\beta_{y 4}}{\beta_{x 4}}-\frac{\beta_{y 3}}{\beta_{x 3}}\right) .
\end{aligned}
$$

Where the rotating angles satisfy

$$
\begin{aligned}
& (-1)^{n_{x 1}+n_{y 1}+n_{x 2}+n_{y 2}} \sqrt{\frac{\beta_{y 3}}{\beta_{x 3}}}\left(\frac{\beta_{y 1}}{\beta_{x 1}}-\frac{\beta_{y 2}}{\beta_{x 2}}\right) \tan 4 \theta_{3}+(-1)^{n_{x 1}+n_{y 1}} \sqrt{\frac{\beta_{y 2}}{\beta_{x 2}}}\left(\frac{\beta_{y 3}}{\beta_{x 3}}-\frac{\beta_{y 1}}{\beta_{x 1}}\right) \tan 4 \theta_{2}+\sqrt{\frac{\beta_{y 1}}{\beta_{x 1}}}\left(\frac{\beta_{y 2}}{\beta_{x 2}}-\frac{\beta_{y 3}}{\beta_{x 3}}\right) \tan 4 \theta_{1}=0, \\
& (-1)^{n_{x 2}+n_{y 2}+n_{x 3}+n_{y 3}} \sqrt{\frac{\beta_{y 4}}{\beta_{x 4}}}\left(\frac{\beta_{y 2}}{\beta_{x 2}}-\frac{\beta_{y 3}}{\beta_{x 3}}\right) \tan 4 \theta_{4}+(-1)^{n_{x 2}+n_{y 2}} \sqrt{\frac{\beta_{y 3}}{\beta_{x 3}}}\left(\frac{\beta_{y 4}}{\beta_{x 4}}-\frac{\beta_{y 2}}{\beta_{x 2}}\right) \tan 4 \theta_{3}+\sqrt{\frac{\beta_{y 2}}{\beta_{x 2}}}\left(\frac{\beta_{y 3}}{\beta_{x 3}}-\frac{\beta_{y 4}}{\beta_{x 4}}\right) \tan 4 \theta_{2}=0 .
\end{aligned}
$$

For the skew octupole case, the strength of the three octupoles must satisfy

$$
(-1)^{n_{x 1}+n_{y 1}} \frac{k_{31} \beta_{x 1}^{2}}{\left(\beta_{y 2} / \beta_{x 2}\right)-\left(\beta_{y 3} / \beta_{x 3}\right)} \sqrt{\frac{\beta_{y 1}}{\beta_{x 1}}}=\frac{k_{32} \beta_{x 2}^{2}}{\left(\beta_{y 3} / \beta_{x 3}\right)-\left(\beta_{y 1} / \beta_{x 1}\right)} \sqrt{\frac{\beta_{y 2}}{\beta_{x 2}}}=(-1)^{n_{x 2}+n_{y 2}} \frac{k_{33} \beta_{x 3}^{2}}{\left(\beta_{y 1} / \beta_{x 1}\right)-\left(\beta_{y 2} / \beta_{x 2}\right)} \sqrt{\frac{\beta_{y 3}}{\beta_{x 3}}} .
$$

\section{SET OF TWO SETUPOLES FOR COUPLED LATTICE}

In Sec. III, we have discussed the different lattice structure which the nonlinearity can be cancelled if the Twiss parameters at magnets, the strength and the rotating angle satisfy some conditions. But there, the transfer matrice (linear map) among the magnets is $2 \times 2$ block diagonal, i.e., the lattice is uncoupled between $x-y$ planes. In this 
section, we discuss the self-cancellation condition for a lattice with strong coupling. The candidate machine can be a Mobius accelerator [10].

Considering a $S_{1}-T_{12}-S_{2}$ channel, where

$$
T_{12}=\left(\begin{array}{cccc}
t_{11} & t_{12} & t_{13} & t_{14} \\
t_{21} & t_{22} & t_{23} & t_{24} \\
t_{31} & t_{32} & t_{33} & t_{34} \\
t_{41} & t_{42} & t_{43} & t_{44}
\end{array}\right)
$$

After passing through this channel, the position of the particle is

$$
\vec{X}_{1}=F\left[2, \theta_{2}, k_{22}, T_{12} F\left(2, \theta_{1}, k_{21}, \vec{X}_{0}\right)\right] .
$$

Expanding Eq. (31), one can have

$$
\begin{aligned}
x_{1}= & t_{11} x_{0}+t_{12} x_{0}^{\prime}+t_{13} y_{0}+t_{14} y_{0}^{\prime}-\frac{k_{21}}{2} x_{0}^{2}\left(t_{12} \cos 3 \theta_{1}-t_{14} \sin 3 \theta_{1}\right) \\
& +k_{21} x_{0} y_{0}\left(t_{14} \cos 3 \theta_{1}+t_{12} \sin 3 \theta_{1}\right)+\frac{k_{21}}{2} y_{0}^{2}\left(t_{12} \cos 3 \theta_{1}-t_{14} \sin 3 \theta_{1}\right), \\
y_{1}= & t_{31} x_{0}+t_{32} x_{0}^{\prime}+t_{33} y_{0}+t_{34} y_{0}^{\prime}-\frac{k_{21}}{2} x_{0}^{2}\left(t_{32} \cos 3 \theta_{1}-t_{34} \sin 3 \theta_{1}\right) \\
& +k_{21} x_{0} y_{0}\left(t_{34} \cos 3 \theta_{1}+t_{32} \sin 3 \theta_{1}\right)+\frac{k_{21}}{2} y_{0}^{2}\left(t_{32} \cos 3 \theta_{1}-t_{34} \sin 3 \theta_{1}\right)
\end{aligned}
$$

Let the nonlinear terms be 0 , one can get

$$
t_{12}=0, \quad t_{14}=0, \quad t_{32}=0, \quad t_{34}=0 .
$$

Since all nonlinear terms in $x_{1}^{\prime}$ and $y_{1}^{\prime}$ must be eliminated, one can get the following equation $(\mathrm{A}[\mathrm{B}]$ means the coefficient of B in A)

$$
\begin{array}{lll}
x_{1}^{\prime}\left[x_{0}^{2}\right]=0, & x_{1}^{\prime}\left[x_{0} y_{0}\right]=0, & x_{1}^{\prime}\left[y_{0}^{2}\right]=0, \\
y_{1}^{\prime}\left[x_{0}^{2}\right]=0, & y_{1}^{\prime}\left[x_{0} y_{0}\right]=0, & y_{1}^{\prime}\left[y_{0}^{2}\right]=0 .
\end{array}
$$

From $x_{1}^{\prime}\left[x_{0}^{2}\right]+x_{1}^{\prime}\left[y_{0}^{2}\right]=0$ and $y_{1}^{\prime}\left[x_{0}^{2}\right]+y_{1}^{\prime}\left[y_{0}^{2}\right]=0$, one can get

$\left(t_{11}^{2}+t_{13}^{2}-t_{31}^{2}-t_{33}^{2}\right) \cos 3 \theta_{2}-2\left(t_{11} t_{31}+t_{13} t_{33}\right) \sin 3 \theta_{2}=0$,

$\left(t_{11}^{2}+t_{13}^{2}-t_{31}^{2}-t_{33}^{2}\right) \sin 3 \theta_{2}-2\left(t_{11} t_{31}+t_{13} t_{33}\right) \cos 3 \theta_{2}=0$.

Introducing two parameters $R$ and $\psi$, one can have

$$
\begin{array}{ll}
t_{11}=R \cos \psi, & t_{31}= \pm R \cos \psi, \\
t_{13}=R \sin \psi, & t_{33}=\mp R \sin \psi .
\end{array}
$$

Further, from Eq. (34), one can solve out

$$
\begin{aligned}
& t_{22}=-\frac{k_{22}}{k_{21}} R^{2} \cos \left(3 \theta_{1} \pm 3 \theta_{2}+2 \psi\right), \\
& t_{24}=\frac{k_{22}}{k_{21}} R^{2} \sin \left(3 \theta_{1} \pm 3 \theta_{2}+2 \psi\right), \\
& t_{42}= \pm \frac{k_{22}}{k_{21}} R^{2} \sin \left(3 \theta_{1} \pm 3 \theta_{2}+2 \psi\right), \\
& t_{44}= \pm \frac{k_{22}}{k_{21}} R^{2} \cos \left(3 \theta_{1} \pm 3 \theta_{2}+2 \psi\right),
\end{aligned}
$$

Substituting Eqs. (33), (36), and (37) into Eq. (30) and using the simpletic condition, one can get

$$
\begin{aligned}
& \theta_{1}=\frac{m \pi}{3} \mp \theta_{2}-\psi, \quad k_{21}=-(-1)^{m} R^{3} k_{22}, \\
& m \in \text { integer, } \quad\left(t_{23} \pm t_{41}\right) \cos \psi=\left(t_{21} \mp t_{43}\right) \sin \psi .
\end{aligned}
$$

Further, Eq. (30) will be reduced to

$$
T_{12}=\left(\begin{array}{cccc}
R C & 0 & R S & 0 \\
t_{21} & \frac{C}{R} & t_{23} & \frac{S}{R} \\
\pm R S & 0 & \mp R C & 0 \\
t_{41} & \pm \frac{S}{R} & t_{43} & \mp \frac{C}{R}
\end{array}\right)
$$

where $C=\cos \psi, S=\sin \psi$, and Eq. (31) will be reduced to

$$
\vec{X}_{1}=T_{12} \vec{X}_{0}
$$

\section{APPLICATION FOR SEXTUPOLE}

In order to understand the analytical equations we give two examples to explain how to apply the theory.

Example 1: two sextupoles' channel application. - Like the $-I$ transformer, the structure in Sec. III A can be used for the chromaticity correction (about chromaticity correction one can see Ref. [11]) to improve the dynamic aperture for storage ring. The example here is a real application in the BEPCII lattice design. Because of the vertical micro- $\beta(1.5 \mathrm{~cm})$, the vertical chromaticity of BEPCII is very large $\left(\xi_{x} / \xi_{y}=-12 /-25\right)$. In the design, two pairs of defocusing sextupoles are used for most of the vertical chromaticity (about 22 units, see Table II) correction to reduce the detuning terms (because of the zero contribution to the detuning terms $d Q_{x} / d \varepsilon_{x}, d Q_{y} / d \varepsilon_{y}, d Q_{y} / d \varepsilon_{x}$ from 
TABLE II. The Twiss functions at R2OS7/R3OS7 and R1IS7/ R4IS7 of BEPCII lattice for the positron ring. The phase advance in both $x$ and $y$ planes between the two sextupoles in each pair is $3 \pi$.

\begin{tabular}{lcc}
\hline \hline & R2OS7/ R3OS7 & R1IS7/ R4IS7 \\
\hline$\beta_{x}(m)$ & 2.890 & 3.143 \\
$\alpha_{x}$ & \pm 1.258 & $\mp 1.748$ \\
$\beta_{y}(m)$ & 17.989 & 18.366 \\
$\alpha_{y}$ & $\mp 4.836$ & $\mp 4.998$ \\
$\eta_{x}(m)$ & 0.5695 & 0.8528 \\
$\eta_{x}^{\prime}{ }_{x}$ & $\mp 0.0312$ & \pm 0.1647 \\
$K_{2} L\left(m^{-2}\right)$ & -5.3 & -5.3 \\
$\Delta \xi_{x}$ & -1.4 & -2.3 \\
$\Delta \xi_{y}$ & 8.7 & 13.3 \\
\hline \hline
\end{tabular}

the two pairs of sextoples), so the dynamic aperture is improved significantly.

Example 2: three sextupoles' channel application.-For three sextupoles' channel, the application is more complicated. This is because the chromaticity contributions of some sextupoles are negative in some cases. For example,

According to Eq. (23), assuming

$$
\begin{aligned}
\theta_{1} & =\theta_{2}=\theta_{3}=0, \quad n_{x 1}=n_{x 2}=1, \\
\frac{\beta_{y 1}}{\beta_{x 1}} & =4.5, \quad \frac{\beta_{y 2}}{\beta_{x 2}}=5, \quad \frac{\beta_{y 3}}{\beta_{x 3}}=5.5,
\end{aligned}
$$

then

$$
2 k_{21} \beta_{x 1}^{3 / 2}=k_{22} \beta_{x 2}^{3 / 2}=2 k_{23} \beta_{x 3}^{3 / 2} .
$$

One can see in this channel, $S_{1}, S_{2}$, and $S_{3}$ can be used for a vertical chromaticity correction. But if interchanging the beta ratio of $S_{1}$ and $S_{2}$,

$$
\frac{\beta_{y 1}}{\beta_{x 1}}=5, \quad \frac{\beta_{y 2}}{\beta_{x 2}}=4.5, \quad \frac{\beta_{y 3}}{\beta_{x 3}}=5.5
$$

then

$$
k_{21} \beta_{x 1}^{3 / 2}=2 k_{22} \beta_{x 2}^{3 / 2}=-2 k_{23} \beta_{x 3}^{3 / 2} .
$$

In this case $S_{3}$ will have an opposite chromaticity contribution with $S_{1}$ and $S_{2}$. Here we assume that the dispersion at $S_{3}$ has the same sign as $S_{1}$ and $S_{2}$. However, this case may be applied in a negative momentum compaction lattice due to both positive and negative dispersion existing in the lattice.

\section{SUMMARY}

In Secs. III and IV, we have discussed different lattice structures (each structure can be called one basic multipole bumper) with which the geometric aberrations of the nonlinear accelerator elements can be fully eliminated for onmomentum particles under thin lens approximation. Actually in order to get more complicated multipole bumper one can arrange the basic multipole bumpers parallel or nested, no any other requirement is necessary among these basic multipole bumpers.

The local correction of nonlinearity can be seen as the case of $n_{x}=n_{y}=0$. But in some cases, the local correction is not feasible, one can apply the multipole bumper to correct the nonlinearity, especially in the interaction region for a collider.

\section{ACKNOWLEDGMENTS}

The author would like to thank Professor A. Chao (SLAC) for his helpful suggestions and discussions.

[1] K. L. Brown, IEEE Trans. Nucl. Sci. 26, 3490 (1979).

[2] R. V. Servranckx and K. L. Brown, IEEE Trans. Nucl. Sci. 26, 3598 (1979).

[3] A. Verdier, Report No. CERN LEP-MA/83-33, 1983.

[4] K. Oide and H. Koiso, Phys. Rev. E 47, 2010 (1993).

[5] M. Donald et al., in Proceedings of the Particle Accelerator Conference, Washington, DC, 1993 (IEEE, Piscataway, NJ, 1993), p. 131.

[6] K. Oide, Nucl. Instrum. Methods Phys. Res., Sect. A 276, 427 (1989).

[7] J. Irwin, Nucl. Instrum. Methods Phys. Res., Sect. A 298, 460 (1990).

[8] O. Napoly, T. M. Taylor, and B. Zotter, Part. Accel. 30, 137 (1990).

[9] J. Irwin et al., in Proceedings of 1991 IEEE Particle Accelerator Conference, edited by L. Lizama and J. Chew (IEEE, New York, 1991), p. 2058.

[10] R. Talman, Phys. Rev. Lett. 74, 1590 (1995).

[11] A. Verdier, Report No. CERN 95-06, 1995, p. 77. 